operativity-that is to say a two-state conformational equilibrium, involving low and high-affinity ( $R$ and $T$ ) forms - have expanded the model by permitting differences between the intrinsic oxygen affinities of the $\alpha$ and $\beta$-chain haems. The further assumption that there is no cooperativity between the ligand binding steps in either state, so long as there is no transition from one to the other, forbids any sequential component in the mechanism.

The choice of numerical parameters to test the model against experimental data is never, of course, wholly selfevident, and admits of a certain degree of sophistry. Ogata and McConnell base their choice of oxygen dissociation constants for the $\alpha$ and $\beta$ chains in the $R$ state on values reported for single chains (concerning which, it must be conceded, there has been some argument). Those for the $T$ state they draw from their own measurements on hybrids, in which either the $\alpha$ or the $\beta$ chains are in the cyanmet-form, and therefore functionally silent. The only other parameter of the model is the allosteric constant, $L$, which governs the position of the equilibrium between the $\mathrm{T}$ and $\mathrm{R}$ conformational states, with all haems unliganded. The effective value of $L$ is modified by the presence of the cofactor, diphosphoglycerate, or of an analogue, because this binds to the $T$ state only and so shifts the equilibrium in its favour by a factor that depends on the cofactor concentration and its (known) binding constant.

Ogata and McConnell use a spinlabelled cofactor analogue, the paramagnetic resonance spectrum of which is sensitive to its precise environment, and indicates that the two hybrid species, with the $\alpha$ and $\beta$ chains respectively in the cyanmet-form, are conformationally indistinguishable, at least by this criterion. With the aid of a value for the parameter $L$, which is set at 3,000 , the system at any given cofactor concentration can be completely described in terms of the balance of $T$ and $\mathbf{R}$ forms, and the degree of saturation of the $\alpha$ and $\beta$ chains in either state. At any given level of oxygen saturation, it turns out that the fractions of molecules in the $\mathbf{R}$ state are always highly, and those in the $\mathrm{T}$ state slightly, saturated, that is to say that the unliganded and fully liganded forms predominate in accordance with the observed cooperativity. In the absence of cofactor, the $T \rightarrow R$ transition occurs largely when the total ligand saturation is about half, whereas in its presence, most of the transition ensues only when three oxygen molecules have been bound. This agrees with indications from several experimental lines. Saturation curves of two abnormal haemoglobins, one an $\alpha$, the other a $\beta$-chain variant, can be fitted if the binding constants in the $T$ states of the abnormal chain, and in particular the values of $L$, are grossly changed, so as to make the $\mathbf{R}$ form accessible in all binding states.

Another $\beta$-chain variant, haemoglobin Kansas, where the replacement occurs at the $\alpha_{1} \beta_{2}$ subunit interface, and so might well be expected to affect the relative energies of the two conformational states, shows the same kind of anomaly in reverse by two very direct criteria. Hopfield, Ogawa and Shulman (Biochem. Biophys. Res. Commun., 49,$1480 ; 1972$ ) have found that the rate constant for carbon monoxide uptake is independent of the degree of saturation, whereas in normal haemoglobin there is an increase in rate, in consequence of the appearance of the rapidly reacting $\mathrm{R}$ form, at a saturation corresponding to nearly three ligand molecules bound. Moreover, Ogawa, Mayer and Shulman (ibid., 1485) find that oxyhaemoglobin and carboxyhaemoglobin Kansas are pulled into the $\mathbf{R}$ form by diphosphoglycerate or its analogues, as reflected by the appearance of features in positions of the proton magnetic resonance spectrum uniquely associated with the deoxygenated condition of the normal protein. This means that, even when oxygenated, the $T$ state is energetically sufficiently accessible to be populated with the aid of the additional stabilization conferred by cofactor binding.

Supporting the many lines of evidence that now point to higher oxygen affinity of the $\alpha$ compared with $\beta$ chains in the $T$ state are experiments of Huestis and Raftery (ibid., 1358), who have introduced a trifluoroacetyl group on cys-93 of the $\beta$ chain. The ${ }^{19} F$ NMR spectrum emanating from this group is very sensitive to events involving the $\beta$ chain. Comparison of the oxygenation-induced shift with spectrophotometric measurements of total oxygen uptake confirms that oxygenation must start at the $\alpha$ chains. Two minuscule dimples in the ${ }^{19} \mathrm{~F}$ NMR spectrum at intermediate stages of oxygenation are interpreted in terms of signifi-

\title{
B Cells Responsive to Phytohaemagglutinin
}

Nor so long ago the thought that lymphocytes could actively proliferate was far from the minds of pathologists and haematologists. When Nowell concluded in 1960 that phytohaemagglutinin (PHA), a protein extracted from seeds of Phaseolus vulgaris, caused active mitosis of human peripheral blood lymphocytes in vitro there was much amazement. Cytogeneticists were the first to take advantage of the result in order to display human chromosomes. Slowly immunologists came to realize that their favourite cell could perform an interesting trick in vitro. Concomitant with this realization came the knowledge that lymphocytes were heterogeneous in relation to their anatomical origins. In 1968 several investigators, working largely with mice, concluded that cells of thymic origin ( $T$ cells), rather than those derived from the bursa or its equivalent in mammals, were the PHA-responsive cells.

With great alacrity this finding was extrapolated to the human species and it was found that in immunodeficiency disease syndromes, which were supposed to involve $T$-cell insufficiency, PHA-responsive lymphocytes were few and far between. The advantages of having an in vitro measure of $\mathrm{T}$ cells in man were enormous and there seemed little reason to suppose that what had been proven beyond doubt in the mouse did not also apply to man. In next Wednesday's Nature New Biology (February 21) Phillips and Roitt present evidence which could prick this bubble of enthusiasm. Specifically the authors suggest that both $B$ and $T$ cells can respond to PHA in man in quite con-

\section{ventional cultural conditions.}

Phillips and Roitt first found that approximately 30 per cent of blast cells in PHA cultures of peripheral blood lymphocytes stain with a fluorescent anti-light chain antiserum. This result suggested either that $T$ cells develop immunoglobulin (Ig) on their surfaces when responding to PHA or that B cells, which are widely thought to have immunoglobulin on their surfaces, could be included among the responsive cells.

When tonsillar lymphocytes were run down columns coated with anti-Ig, the resulting $T$ cell-rich population was transformed with PHA in vitro, but as few as 5 per cent of staining cells could be counted. By using dextranase it was possible to recover the retained $B$ cells from the column coated with anti-Ig and these gave rise to 86 per cent staining blasts when cultured with PHA. Incidentally no controls are quoted in which dextranase was used to treat $\mathrm{T}$ cells to see if they then developed immunoglobulin on their surfaces. But aside from this peccadillo the evidence of Phillips and Roitt strongly suggests that $B$ cells in man can transform to PHA and that PHA transformation is thus an unreliable criterion of T-cell numbers in the human species.

It seems that care must be taken in attributing any characteristic as an absolute marker of either $\mathrm{T}$ or $\mathrm{B}$ cells and that extrapolation between species in relation to the distinguishing features of the two cells is hazardous. It should be noted that this argument applies to the use of surface immunoglobulin as a criterion of the B cell. 